\title{
User Tasks Description: a Retrospective, Recent Contributions and some Research Challenges
}

\author{
Philippe Palanque \\ ICS-IRIT, Université Toulouse \\ III - Paul Sabatier \\ 118, route de Narbonne, 31042 \\ Toulouse, France \\ palanquedirit.fr
}

DOI: 10.37789/rochi.2020.1.1.1

\begin{abstract}
Describing users tasks has been the focus of research for many years starting with the seminal work from Annett and Duncan in 1967 [2]. Since then, the Human Factors and Human Computer Interaction domains have proposed multiple contributions identifying the elements that have to be gathered and represented in order to describe precisely the relevant aspects of users tasks. This keynote will highlight these fundamental elements of tasks descriptions and will state the current state of the art. A specific view on how to use such descriptions to design and assess automation will be given. Some publicly available tools will also be presented together with their use in various industrial application domains. These applications will be the opportunity to identify remaining research challenges for tasks description and modeling.
\end{abstract}

\section{Author Keywords}

Task descriptions; task modeling; task-centered design; task-centered evaluation.

ACM Classification Keywords

CCS Concepts: - Human-centered computing $\rightarrow$ Human computer interaction (HCI); Interactive Systems and Tools

\section{HISTORICAL PERSPECTIVE}

Tasks Analysis and Tasks Representation are cornerstones of User Centered Design approaches, aiming to collect information from users goals, work and activities. According to Johnson [16] "any Task Analysis is comprised of three major activities; first, the collection of data; second, the analysis of that data; and third, the modelling of the task domain" (p.165). While this work emphasises the importance of data, it is important to note that users' work is mainly a procedural activity. The means (notations and tools for representing the outcomes of task analysis) have important implications for the value and insight gained from the process, not least because any omissions cannot be discussed (among the stakeholders) or taken into consideration in later design phases.

The expressive power of the notation used to store and organize the information collected is thus a key element that is put forward by researchers proposing new notations [4]. Since the seminal HTA notation proposed by [1][2], relatively few notations to describe user tasks have been proposed and they all tend to remain unchanged after their creation. While the notations remain largely constant, their associated tools typically evolve to address new challenges. For instance, Paterno's team has proposed several tools exploiting CTT notation since its creation in 1997 [35]: the original CTTe tool Error! Reference source not found. supported editing, simulating and verifying CTT task models; CTTEVis [36] added support for visualization; and a new tool added support for collaborative modelling [20]. The stability of notations contrasts with the constant evolution of application domains, technologies, and the nature of work operators' work. This evolution can create a gap between what the notation can describe and the actual work, limiting the scope and potential benefits of using the notation at all. For instance, a notation like KMAD [4] or CTT [35] would produce the same representation of tasks for interacting with a calculator regardless of whether the calculator was a physical device, a desktop application, or an app on a mobile phone. This lack of precision and detail make it impossible for analysts to assess the interaction implications of moving from one technology to the other one.

This is heavily constrated with the work on the HAMSTERS notation that was designed after the fundamental elements of users' tasks descriptions were identified and incorporated in notations.

HAMSTERS was built exploiting these fundamental elements that are:

- Hierarchical structure of tasks: Hierarchical Task Analysis [1] [2],

- Knowledge representation: Task Knowledge Structure [17],

- Multi-user/Collaborative activities: Groupware Task Analysis [40].

In order to address the challenges brought by new technologies, for each of the fundamentals elements above, HAMSTERS was extended to represent much more precise information:

- Tasks structuring using sub models [27] and components [10] 
- Detailed knowledge and information representation [26] ;

- Synchronous, asynchronous, same place, different place collaboration [21].

More rencently, HAMSTERS notation and its associated tool HAMSTERS|XL have been released to allow human factors analysts to customize the HAMSTERS notation to the needs of the application domain or the interactive systems they are addressing. For instance, in [8] the HAMSTERS notation was extended to address the specificities of Cyber Physical Systems.

\section{EXPLOITING TASKS DESCRIPTIONS}

The following non-exhaustive list identifies some of the objectives of task analysis, highlighting its broad range of uses:

- Identification and description of the required functions for interactive system [13] [35],

- Identification and description of knowledge required to perform a task [7] [17] [26] [38],

- Identification and description of the temporal ordering of the user actions with the system [18] [22] [35],

- Identification and description of the different user roles and actors for groupware systems [37] [40],

- Identification and description of workflow between users for collaborative activities [37] [40],

- $\quad$ Understanding of an application domain [34],

- Recording the results of interdisciplinary discussions [30] [34],

- Production of scenarios for user evaluation [41] as well identification and generation of relevant test cases [6],

- Heuristic evaluation of usability of interactive applications [5] [37],

- Predictive assessment of task complexity and workload (motor, cognitive, perceptive) [29],

- Predictive assessment of user performance when interacting with the system [15],

- Exploration of the range of ways in which the system may be used [37],

- Preparation of training programs [1] [2] [25],

- Production of user manual [12] [33] and contextual help [14] [31] [33] [34],

- Identification and description of possible allocation of functions and tasks between the system and the user [23] [32],

- Designing new applications consistent with the user conceptual model [34],

- Identification and description of potential user errors [9] [39] [42] [24],

- Assessing other properties than usability as, for instance, dependability [43], security [44] or user experience [45].

Task analysis is thus a pillar of UCD approaches for the design of interactive systems. If the results of task analysis do not contain sufficient information, the missing information may negatively affect the design of the interactive system and its usability.

\section{CHALLENGES AHEAD}

The main challenge ahead of users' tasks representation lays in the fact that there is reluctance to use this tool that requires deep understanding and description of users' work.

Beyond, curricula like the ACM 2013 Computer Science curricula that exhibits multiple courses on Human-Computer Interaction does not even mention the need to analyse and represent users' tasks [3]. There is still a long road ahead before seing widespread use of task modeling noations and tools but the path created by CTTE which offered the first publicly available tool increased the takeup-ability of the multiple benefits of users' tasks representation.

\section{REFERENCES}

[1] John Anett. 2004. Hierarchical Task Analysis. In Diaper Dan, Stanton Neville (Eds), The Handbook of Task Analysis for Human-Computer Interaction (pp. 67-82). Lawrence Erlbaum Associates.

[2] John Annett, Keith Duncan.1967. Task analysis and training design. Occupational psychology, 41, 211-221.

[3] ACM CS curriculum 2013 https://www.acm.org/binaries/content/assets/education/cs2013 web final.pdf

[4] Sybille Caffiau, Dominique Scapin, Patrick Girard, Mickaël Baron, and Francis Jambon. 2010. Increasing the expressive power of task analysis: Systematic comparison and empirical assessment of toolsupported task models. Interact. with Comput. 22, 6 (November 2010), 569-593.

[5] Cockton, G., \& Woolrych, A. (2001). Understanding inspection methods: Lessons from an assessment of heuristic evaluation. People and Computers XV, joint Proceedings of HCI 2001 and IHM 2001, Springer Verlag 171-192.

[6] José Creissac Campos, Camille Fayollas, Marcelo Gonçalves, Célia Martinie, David Navarre, Philippe Palanque, and Miguel Pinto. 2017. A More Intelligent Test Case Generation Approach through Task Models Manipulation. Proc. ACM Hum.-Comput. Interact. 1, EICS, Article 9 (June 2017), 20 pages

[7] Dan Diaper. 1990. Task Analysis for Knowledge Descriptions (TAKD): The Method and an Example. In Task Analysis for HumanComputer Interaction, D. Diaper (ed.), Ellis Horwood, pp. 108-159.

[8] R. Fahssi, C. Martinie and P. Palanque, "Embedding explicit representation of cyber-physical elements in task models," 2016 IEEE International Conference on Systems, Man, and Cybernetics (SMC), Budapest, 2016, pp. 001969-001974, doi: 10.1109/SMC.2016.7844528.

[9] Racim Fahssi, Celia Martinie, Philippe Palanque. 2015. Enhanced Task Modelling for Systematic Identification and Explicit Representation of Human Errors. In: Abascal J., Barbosa S., Fetter M., Gross T., Palanque P., Winckler M. (eds) IFIP TC13 Conference on Human-Computer Interaction - INTERACT 2015. Lecture Notes in Computer Science, vol 9299.

[10] Peter Forbrig, Célia Martinie, Philippe Palanque, Marco Winckler, and Racim Fahssi. 2014. Rapid Task-Models Development Using Sub-models, Sub-routines and Generic Components. In Proceedings of the 5th IFIP WG 13.2 International Conference on HumanCentered Software Engineering - Volume 8742 (HCSE 2014) Vol. 8742. Springer-Verlag, 144-163.

[11] Matthias Giese, Tomasz Mistrzyk, Andreas Pfau, Gerd Szwillus, and Michael Detten. 2008. AMBOSS: A Task Modelling Approach for Safety-Critical Systems. In Proceedings of the 2nd Conference on Human-Centered Software Engineering and 7th International Workshop on Task Models and Diagrams (HCSE-TAMODIA '08), Springer-Verlag, Berlin, Heidelberg, 98-109. 
[12] Gong, R. \& Elkerton, J. (1990). Designing minimal documentation using the GOMS model: A usability evaluation of an engineering approach. CHI 90 Proceedings. New York, ACM DL.

[13] Saul Greenberg. Working through Task-Centered System Design. In Diaper, D. and Stanton, N. (Eds) The Handbook of Task Analysis for Human-Computer Interaction. Lawrence Erlbaum Associates (2004). p49-66.

[14] Valeria Gribova. A method of Context-Sensitive Help Generation Using a Task Project. International Journal on Information Theories \& Applications Vol.15, pp. 391-395, 2008.

[15] Bonnie E. John and David E. Kieras. 1996. The GOMS family of user interface analysis techniques: comparison and contrast. ACM Trans. Comput.-Hum. Interact. 3, 4 (December 1996), 320-351.

[16] Peter Johnson. 1992. Human-Computer Interaction: psychology, task analysis and software engineering, McGraw Hill, Maidenhead, UK.

[17] Peter Johnson, H. Johnson and F. Hamilton. 2000. Getting the Knowledge into HCI: Theoretical and Practical Aspects of Task Knowledge Structures. In. Cognitive Task Analysis. J. Schraagen, S. Chipman, V. Shalin LEA

[18] Frédéric Jourde, Yann Laurillau, and Laurence Nigay. 2010. COMM notation for specifying collaborative and multimodal interactive systems. In Proceedings of the 2nd ACM SIGCHI symposium on Engineering interactive computing systems (EICS '10). ACM, New York, NY, USA, 125-134.

[19] James Lin, Mark W. Newman, Jason I. Hong, and James A. Landay. 2000. DENIM: finding a tighter fit between tools and practice for Web site design. In Proceedings of the SIGCHI conference on Human Factors in Computing Systems (CHI '00). ACM, New York, NY, USA, 510-517.

[20] Marco Manca, Fabio Paternò, Carmen Santoro. 2016. Collaborative Task Modelling on the Web. In: Bogdan C. et al. (eds) HumanCentered and Error-Resilient Systems Development. HESSD 2016, HCSE 2016. Lecture Notes in Computer Science, vol 9856. Springer,.

[21] Célia Martinie, Eric Barboni, David Navarre, Philippe Palanque, Racim Fahssi, Erwann Poupart, and Eliane Cubero-Castan. 2014. Multi-models-based engineering of collaborative systems: application to collision avoidance operations for spacecraft. In Proceedings of the 2014 ACM SIGCHI symposium on Engineering interactive computing systems (EICS '14). ACM, USA, 85-94.

[22] Célia Martinie, David Navarre, Philippe Palanque, and Camille Fayollas. 2015. A generic tool-supported framework for coupling task models and interactive applications. In Proceedings of the 7th ACM SIGCHI Symposium on Engineering Interactive Computing Systems (EICS '15). ACM, New York, NY, USA, 244-253.

[23] Célia Martinie, Philippe Palanque, Eric Barboni and Martina Ragosta. 2011. Task-model based assessment of automation levels: Application to space ground segments. IEEE International Conference on Systems, Man, and Cybernetics, pp. 3267-3273.

[24] Célia Martinie, Philippe A. Palanque, Racim Fahssi, Jean-Paul Blanquart, Camille Fayollas, Christel Seguin. 2016. Task ModelBased Systematic Analysis of Both System Failures and Human Errors. IEEE Trans. Human-Machine Systems 46(2), 243-254.

[25] Célia Martinie, Philippe Palanque, David Navarre, Marco Winckler, and Erwann Poupart. 2011. Model-based training: an approach supporting operability of critical interactive systems. In Proceedings of the 3rd ACM SIGCHI symposium on Engineering interactive computing systems (EICS '11). ACM, New York, NY, USA, 53-62.

[26] Célia Martinie, Philippe Palanque, Martina Ragosta, and Racim Fahssi. 2013. Extending procedural task models by systematic explicit integration of objects, knowledge and information. In Proceedings of the 31st European Conference on Cognitive Ergonomics (ECCE '13). ACM, Article 23, 10 pages.

[27] Célia Martinie, Philippe Palanque, and Marco Winckler. 2011. Structuring and composition mechanisms to address scalability issues in task models. In Proceedings of the 13th IFIP TC 13 international conference on Human-computer interaction - Volume Part III (INTERACT'11), Pedro Campos, Nuno Nunes, Nicholas Graham, Joaquim Jorge, and Philippe Palanque (Eds.), Vol. Part III. SpringerVerlag, Berlin, Heidelberg, 589-609.

[28] Célia Martinie, Philippe Palanque, Elodie Bouzekri, Andy Cockburn, Alexandre Canny, and Eric Barboni. 2019. Analysing and Demonstrating Tool-Supported Customizable Task Notations. Proc. ACM Hum.-Comput. Interact. 3, EICS, Article 12 (June 2019), 26 pages. DOI:https://doi.org/10.1145/3331154

[29] O'Donnell, R. D.; Eggemeier, F. T. Workload Assessment Methodology; In K. R. Boff \& L. Kaufman \& J. P. Thomas (Eds.), Handbook of Perception and Human Performance (Vol. II Cognitive Processes and Performance, pp. 42-41 - 42-49). Wiley \& Sons, 1986.

[30] Eamonn O'Neill and Peter Johnson. 2004. Participatory task modelling: users and developers modelling users' tasks and domains. In Proceedings of the 3rd annual conference on Task models and diagrams (TAMODIA '04). ACM, New York, NY, USA, 67-74.

[31] Philippe Palanque, Rémi Bastide, Louis Dourte. Contextual Help for Free with Formal Dialogue Design. In Proc. of HCI International 1993.

[32] Philippe Palanque, Célia Martinie, and Camille Fayollas. 2018. Automation: Danger or Opportunity? Designing and Assessing Automation for Interactive Systems. In Extended Abstracts of the 2018 CHI Conference on Human Factors in Computing Systems (CHI EA '18). ACM, New York, NY, USA, Paper C19, 4 pages.

[33] Pangoli S., Paternò F. Automatic Generation of Task-Oriented Help. ACM Symposium on UIST 1995, 181-187.

[34] Fabio Paterno. Task models in interactive software systems, Handbook of Software Engineering and Knowledge Engineering, Vol 1, 2002, Publisher: World Scientific, pp. 1-19.

[35] Fabio Paternò, Cristiano Mancini, Silvia Meniconi. ConcurTaskTrees. 1997. A Diagrammatic Notation for Specifying Task Models. In Proc. of IFIP INTERACT 1997, pp. 362-369.

[36] Fabio Paternò and Enrico Zini. 2004. Applying information visualization techniques to visual representations of task models. In Proceedings of the 3rd annual conference on Task models and diagrams (TAMODIA '04). ACM, New York, NY, USA, 105-111.

[37] David Pinelle, Carl Gutwin, and Saul Greenberg. 2003. Task analysis for groupware usability evaluation: Modelling shared-workspace tasks with the mechanics of collaboration. ACM Trans. Comput.Hum. Interact. 10, 4 (December 2003), 281-311.

[38] Martina Ragosta, Célia Martinie, Philippe Palanque, David Navarre, and Mark Alexander Sujan. 2015. Concept Maps for Integrating Modelling Techniques for the Analysis and Re-Design of PartlyAutonomous Interactive Systems. In Proc. of the 5th International Conference on Application and Theory of Automation in Command and Control Systems (ATACCS '15), ACM, 41-52.

[39] Daniel Sinnig, Maik Wurdel, Peter Forbrig, Patrice Chalin, Ferhat Khendek. Practical Extensions for Task Models. In proc. of TAMODIA 2007, 42-55, Springer.

[40] Gerrit C. van der Veer, Bert F. Lenting, Bas A.J. Bergevoet. 1996 GTA: Groupware task analysis - Modelling complexity, Acta Psychologica, Volume 91, Issue 3, pages 297-322.

[41] Marco Winckler, Philippe Palanque, and Carla M. D. S. Freitas. 2004. Tasks and scenario-based evaluation of information visualization techniques. In Proceedings of the 3rd annual conference on Task models and diagrams (TAMODIA '04). ACM, New York, NY, USA, 165-172.

[42] Palanque, P., Basnyat, S.: Task Patterns For Taking Into Account In An Efficient and Systematic Way Both Standard And Erroneous User Behaviours. In: IFIP 13.5 Working Conf. on Human Error, Safety and Systems Development (HESSD), pp. 109-130. Kluwer Academic Publisher, Dordrecht (2004)

[43] C. Fayollas, C. Martinie, P. Palanque, Y. Deleris, J. -. Fabre and D. Navarre, "An Approach for Assessing the Impact of Dependability on Usability: Application to Interactive Cockpits," 2014 Tenth European Dependable Computing Conference, Newcastle, 2014, pp. 198-209, doi: 10.1109/EDCC.2014.17.

[44] Nicolas Broders, Célia Martinie, Philippe Palanque and Kimmo Halunen. A Generic Multimodels-Based Approach for the Usability and Security Analysis of Authentication Mechanisms. 8th International Conference on Human-Centered Software Engineering 2020, LNCS, Springer, to appear

[45] Bernhaupt R., Palanque P., Drouet D., Martinie C. (2019) Enriching Task Models with Usability and User Experience Evaluation Data. In: Bogdan C., Kuusinen K., Lárusdóttir M., Palanque P., Winckler M. (eds) Human-Centered Software Engineering. HCSE 2018. 
Proceedings of RoCHI 2020

Lecture Notes in Computer Science, vol 11262. Springer, Cham. https://doi.org/10.1007/978-3-030-05909-5_9 